\title{
AN ALGORITHMIC ANALYSIS OF THE INTERSECTION PROPERTY
}

\section{PASCALE JACOBS AND DIMITRI LEEMANS}

\begin{abstract}
In this paper, efficient algorithms are given to test the intersection property and some of its variations on flag-transitive coset geometries. These algorithms are then applied to geometries of some sporadic groups, namely the Mathieu groups $\mathbf{M}_{11}, \mathbf{M}_{12}, \mathbf{M}_{22}$ and $\mathbf{M}_{23}$, the Janko groups $\mathbf{J}_{1}, \mathbf{J}_{2}$ and $\mathbf{J}_{3}$ and the Higman-Sims group HS.
\end{abstract}

\section{Introduction}

In incidence geometry, the main question that is still open nowadays is, as mentioned by Mauro Biliotti and Antonio Pasini in [1]: 'What are the right axioms for good geometries?'

In [9], a synthesis of the work that has been going on in that direction for the last fifteen years is presented, and a set of axioms is given. Such work has been heavily based on computer experiments; see [9] for a bibliography on the subject. We refer the reader also to the paper by Michel Dehon [11], where efficient algorithms to test the flag-transitivity of a geometry are given, and the paper by Dehon and Leemans [13], which describes two algorithms that can be used to obtain classifications of geometries that satisfy the axioms of [9]. There is a need to test new properties on the lists of geometries that have already been obtained, in order to attempt to extend the list of axioms given in [9].

The intersection property (IP) has been extensively studied for a long time. It had already appeared in the work of Jacques Tits as early as 1956 (see [23]), even before the birth of geometric buildings. It is one of the most important properties in incidence geometry.

As Francis Buekenhout pointed out in [7], it is often tedious to check this property in full detail for geometries over a non-linear diagam. However, the (IP) was contained in the axioms that Buekenhout imposed on his geometries from the beginning [3], although for technical reasons it was not initially included in the set of axioms used by Buekenhout and Dehon when they started their experimental work.

All these arguments show that it is important to have an efficient algorithm to test the intersection property.

There are several versions of this property, as shown in the paper by Biliotti and Pasini [1], and later on by Pasini in [22]. We decided to investigate (and we describe in this paper) the version that seems to be the most commonly used. This was introduced by Buekenhout in [3], and is the property referred to as the (IP) in reference [6] (see also, for instance, $[\mathbf{4 , 5}]$ for nice applications of this property). We have also studied some weakened forms of the property, namely the properties (IP) ${ }_{n},(\mathrm{WIP})_{n}$ and (WIP). Our motivation was that the condition (IP) might be too strong to include in the set of axioms of [9]; we note that the (IP) 2 has already been included in these axioms. Moreover, it is easy to derive algorithms to test these weakened forms from the algorithm that is used to test the (IP). 
In this paper, we describe an algorithm to test the intersection property and some of its variants on a flag-transitive coset geometry. We implemented this algorithm using the computer algebra package MAGMA [2]. We then used this program to test the above properties on a series of geometries for the Mathieu groups $\mathrm{M}_{11}, \mathrm{M}_{12}, \mathrm{M}_{22}$ and $\mathrm{M}_{23}$, the Janko groups $\mathrm{J}_{1}, \mathrm{~J}_{2}$ and $\mathrm{J}_{3}$, and the Higman-Sims group HS. This shows that the algorithm described in this paper may be used on rather large geometries (that is, geometries with more than one million elements).

The paper is organised as follows. In Section 2, we recall some definitions and fix the notation. In Section 3, we describe algorithms to test both the intersection property itself and some weaker forms of it. We apply these algorithms in Section 4 to lists of geometries for the eight smallest sporadic simple groups. In Section 5, we mention some results obtained for the Suzuki group $\mathrm{Sz}(8)$, and for inductively minimal geometries. Finally, in Section 6, we give the programs that we used to obtain the results given in Section 4. These programs are written in MAGMa.

There are two electronic appendices to the paper. The source code given in Section 6 is provided as a downloadable text file in Appendix A. This file can be loaded in MAGMA using the 'load' command. Appendix B is a gzipped tar archive of text files. These text files contain the maximal parabolic subgroups of the geometries for the eight smallest sporadic groups mentioned in Section 4. The archive also contains a README file, which explains how to use the files in Magma.

\section{Definitions and notation}

We assume knowledge of the basic notions in incidence geometry, as given, for instance, in $[8]$ or $[22]$.

Let $\Gamma(X, *, t, I)$ be an incidence geometry. Given a type $i \in I$, for any flag $F$ of $\Gamma$ we define the $i$-shadow $\sigma_{i}(F)$ as the set of elements of type $i$ incident with $F$.

We define the intersection property (IP) as it appears in [3].

(IP) For every type $i$, the intersection of the $i$-shadows of a variety $x$ and a flag $F$ is empty, or it is the $i$-shadow of a flag incident to $x$ and $F$. The same holds on the residues.

In earlier work, the second author, together with co-workers including Francis Buekenhout, Michel Dehon and Philippe Cara, imposed a condition denoted by $(\mathrm{IP})_{2}$. This condition requires that all rank-2 residues of $\Gamma$ satisfy the (IP). If $\Gamma$ is a geometry of rank $n$, we could define a property $(\mathrm{IP})_{k}$ in the following way (for $k=2, \ldots, n$ ), as suggested by Francis Buekenhout.

$(\mathrm{IP})_{k}$ For every residue $\mathcal{R}$ of rank $k$ of $\Gamma$, for every type $i$ in the set of types of $\mathcal{R}$, the intersection of the $i$-shadows of a variety $x$ and a flag $F$ is empty, or it is the $i$-shadow of a flag incident to $x$ and $F$.

The following lemma is obvious.

Lemma 2.1. Let $\Gamma$ be a geometry of rank $n$. Then $\Gamma$ has the (IP) if and only if $\Gamma$ has the $(\mathrm{IP})_{k}$ for all $k \in\{2, \ldots n\}$.

We may weaken the (IP) $)_{k}$ property in the following way.

(WIP) $k$ For every residue $\mathcal{R}$ of rank $k$ of $\Gamma$, there exists a type $i$ in the set of types of $\mathcal{R}$ such that the intersection of the $i$-shadows of a variety $x$ and a flag $F$ is empty, or it is the $i$-shadow of a flag incident to $x$ and $F$.

Moreover, as in the definition of the (IP), we define the (WIP) as follows. 
(WIP) For at least one type $i$, the intersection of the $i$-shadows of a variety $x$ and a flag $F$ is empty, or it is the $i$-shadow of a flag incident to $x$ and $F$. The same holds on the residues.

Again, the following lemma is obvious.

LemMa 2.2. Let $\Gamma$ be a geometry of rank $n$. Then $\Gamma$ has the (WIP) if and only if $\Gamma$ has the $(\mathrm{WIP})_{k}$ for all $k \in\{2, \ldots n\}$.

\section{Algorithms}

The definitions of the properties (IP) ${ }_{k},(\mathrm{WIP})_{k},(\mathrm{IP})$ and (WIP) may be easily translated into programs. The problem is that we would like these properties to be checkable on rather large geometries - for instance, on geometries with about a million elements. The geometries that we are interested in are constructed from groups by using Tits' algorithm, which we now recall.

THEOREM 3.1 (Tits' algorithm [24]). Let I be a finite set. Let $G$ be a group, and let $\left(G_{i}\right)_{i \in I}$ be a collection of subgroups of $G$. Let

$X=\left\{G_{i} g \mid i \in I, g \in G\right\} ; \quad t: X \rightarrow I: G_{i} g \rightarrow i ; \quad *=\left\{\left(G_{i} g, G_{j} h\right) \mid G_{i} g \cap G_{j} h \neq \emptyset\right\}$.

Then $\Gamma=\Gamma\left(G ;\left(G_{i}\right)_{i \in I}\right)=(X, *, t, I)$ is an incidence structure having a chamber. Moreover, $G$ acts by right multiplication as an automorphism group of $\Gamma$. Finally, $G$ is transitive on the flags of rank at most 2.

When $\Gamma$ is constructed using Tits' algorithm, we say that $\Gamma$ is a coset geometry. Most of the time, we are interested in flag-transitive coset geometries. These are geometries $\Gamma\left(G ;\left(G_{i}\right)_{i \in I}\right)$ on which the group $G$ acts flag-transitively. We describe an algorithm to test the properties $(\mathrm{WIP})_{k}$ and $(\mathrm{IP})_{k}$ on a flag-transitive coset geometry

$$
\Gamma:=\Gamma\left(G ;\left(G_{i}\right)_{i \in\{1, \ldots n\}}\right) \quad \text { of rank } n \geqslant k \geqslant 3 .
$$

When $k=2$, it is well known that $\Gamma$ has (IP $)_{2}$ if and only if all its rank-2 residues are either partial linear spaces or generalized digons. Therefore, this property can be easily checked just by looking at the diagram of $\Gamma$.

If $\Gamma$ is a geometry of rank $n>k$, we test property (IP) $k$ on $\Gamma$ by checking that all residues of rank $k$ of $\Gamma$ satisfy the (IP) $k$. We may thus assume without loss of generality that $\Gamma$ is a geometry of rank $k$.

We must check that for every type $i \in I=\{1, \ldots k\}$, for every element $x$ and for every flag $F$, if $\sigma_{i}(x) \cap \sigma_{i}(F) \neq \emptyset$, then there exists a flag $F^{\prime}$ such that $F^{\prime}$ is incident to $x$ and $F$ and $\sigma_{i}(x) \cap \sigma_{i}(F)=\sigma_{i}\left(F^{\prime}\right)$. We make some observations that will help lead us to an efficient algorithm.

- Since $\Gamma$ is flag-transitive, we have only to check that property for one element $x$ of each type.

- Moreover, we may assume that $x$ is not of type $i$, for otherwise the property is obviously true. Similarly, we may assume that $i \notin t(F)$.

- If $x$ and $F$ are such that $\sigma_{i}(x) \cap \sigma_{i}(F) \neq \emptyset$, then there exists an element of type $i$, say $\infty$, incident to both $x$ and $F$. Thus $x$ and $F$ belong to the residue of $\infty$ in $\Gamma$.

- Since $\Gamma$ is flag-transitive, we may assume that $\infty$ is $G_{i}$ and that $x$ is $G_{j}$.

- We may assume that $F$ is not the empty flag; otherwise the property is obviously true.

- Moreover, the flags $F$ for which we must check the property are in the residue of $G_{i}$ in $\Gamma$. 


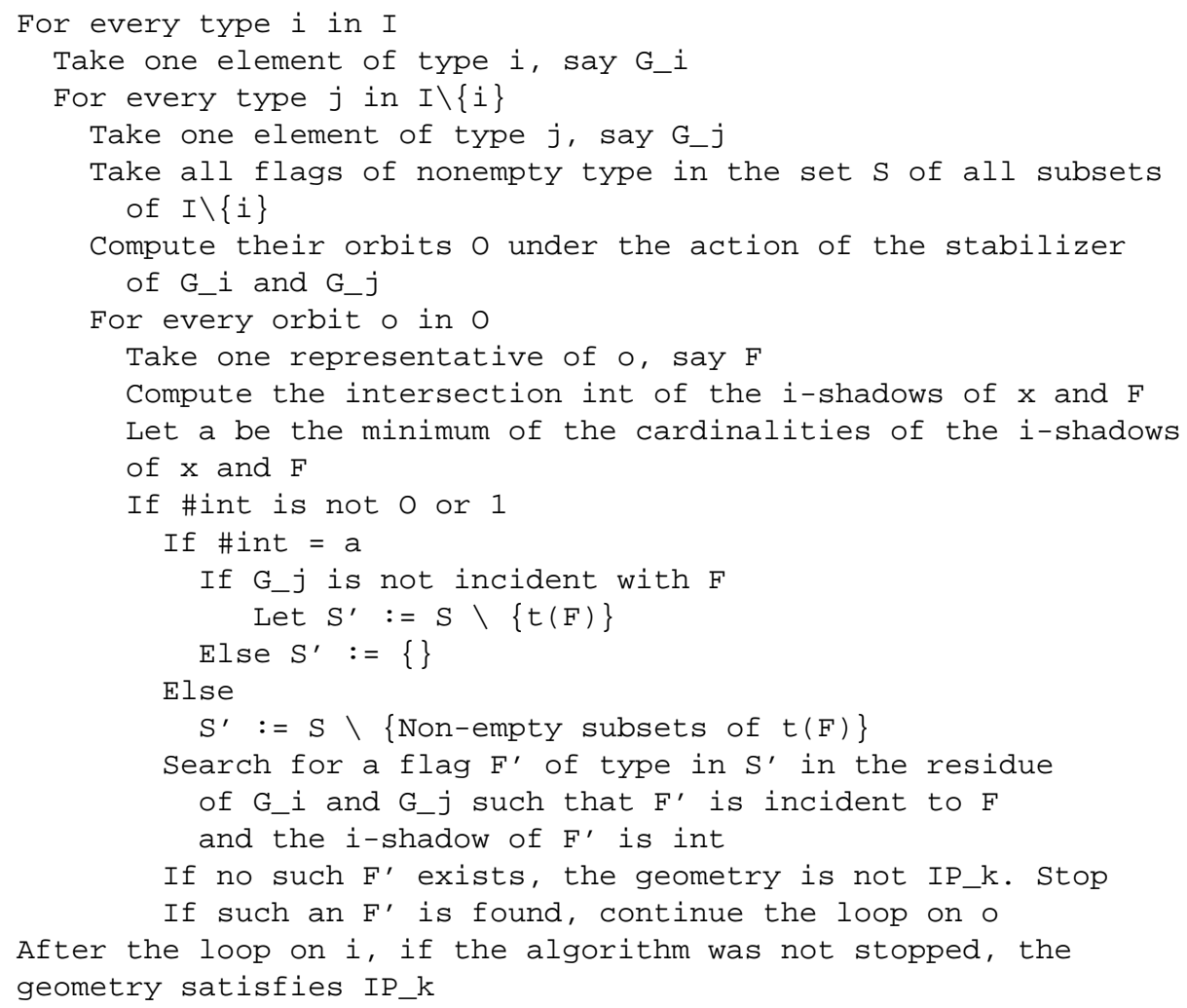

Figure 1: An algorithm to test whether a geometry $\Gamma$ of rank $k$ satisfies the $(\mathrm{IP})_{k}$.

- We may compute the action of the stabilizer of $\infty$ and $x$ in $G$ and its orbits on the flags $F$ of type a subset of $I \backslash\{i\}$. Then, for each orbit, we take one representative $F$ and compute its $i$-shadow $\sigma_{i}(F)$.

- Let $\alpha=\left|\sigma_{i}(x) \cap \sigma_{i}(F)\right|$. It is obvious that $0 \leqslant \alpha \leqslant \min \left(\left|\sigma_{i}(x)\right|,\left|\sigma_{i}(F)\right|\right)$.

- If $\alpha=0$, then $x$ and $F$ have non-intersecting $i$-shadows, and nothing further needs to be done.

- If $\alpha=1$, then $x$ and $F$ are incident to a same element $y$ of type $i$ and $\sigma_{i}(x) \cap \sigma_{i}(F)=$ $\sigma_{i}(y)$.

- If $\alpha=\min \left(\left|\sigma_{i}(x)\right|,\left|\sigma_{i}(F)\right|\right)$ and $x$ is incident with $F$, then we take $F^{\prime}=\{x\}$ or $F$, depending on whether or not $\alpha=\left|\sigma_{i}(x)\right|$.

- Thus it is only:

(i) when $1<\alpha<\min \left(\left|\sigma_{i}(x)\right|,\left|\sigma_{i}(F)\right|\right)$, or

(ii) when $\alpha=\min \left(\left|\sigma_{i}(x)\right|,\left|\sigma_{i}(F)\right|\right)$ and $x$ and $F$ are not incident,

that we have to search for $F^{\prime}$. Moreover, in the first case, we know that if $t\left(F^{\prime}\right) \subseteq t(F)$, then $\left|\sigma_{i}\left(F^{\prime}\right)\right| \geqslant\left|\sigma_{i}(F)\right|$. Therefore we need only to look for flags $F^{\prime}$ of type $t\left(F^{\prime}\right)$ such that $t\left(F^{\prime}\right) \subseteq I \backslash\{i\}$ and $t\left(F^{\prime}\right) \nsubseteq t(F)$. In the second case, we may assume that $t\left(F^{\prime}\right) \neq t(F)$, for otherwise this would imply that $F=F^{\prime}$, and $F^{\prime}$ would not be incident with $x$.

Figure 1 gives an algorithm that follows from the above observations. In this algorithm, we describe how to check for property $(\mathrm{IP})_{k}$ on a geometry of rank $k$. One may easily 
change the algorithm in order to check for the (WIP) $)_{k}$. Indeed, it would suffice in that case to find one value of $i$ for which the property is satisfied.

\section{Results for sporadic groups}

In this section, we give the results that were obtained on lists of geometries for sporadic groups, using our programs. The lists that we decided to look at are those of residually weakly primitive and $(2 T)_{1}$ geometries for the eight smallest sporadic groups. We tested for the properties (WIP) $)_{i}$ and (IP) $)_{i}$ on geometries that are available in [14], [12], [18], [20], [15], [19] and [21], for the groups $\mathrm{M}_{11}, \mathrm{M}_{12}, \mathrm{M}_{22}, \mathrm{M}_{23}, \mathrm{~J}_{1}, \mathrm{~J}_{2}, \mathrm{~J}_{3}$ and $\mathrm{HS}$, respectively. The geometries listed in this section are available in Appendix B of this paper. The results obtained are summarized in Tables 1-8.

Tables 1 and 2 summarize the results for the (IP) 3 and (WIP) 3 properties. In Table 1 , we give, for a group $G$ and for a given rank $r$ :

- the number of geometries of $\operatorname{rank} r$ that $G$ has (This is the number of geometries that are firm, residually connected and (IP) $)_{2}$, and on which the group $G$ acts flag-transitively, residually weakly primitively and locally 2-transitively; see [9] for definitions.);

- how many of them satisfy, respectively: (i) the (IP) $)_{3}$, or (ii) the (WIP) $)_{3}$ but not the $(\mathrm{IP})_{3}$, and how many do not satisfy the (WIP) $)_{3}$.

Table 1: Number of geometries that are $(\mathrm{IP})_{3},(\mathrm{WIP})_{3}$ but not $(\mathrm{IP})_{3}$, and not $(\mathrm{WIP})_{3}$.

\begin{tabular}{|c|c|c|c|c|c|}
\hline Group & Rank & \# of geometries & $(\mathrm{IP})_{3}$ & $(\mathrm{WIP})_{3}$ but not $(\mathrm{IP})_{3}$ & not $(\mathrm{WIP})_{3}$ \\
\hline \multirow[t]{2}{*}{$\mathrm{M}_{11}$} & 3 & 13 & 6 & 1 & 6 \\
\hline & 4 & 11 & 2 & 0 & 9 \\
\hline \multirow[t]{3}{*}{$\mathrm{M}_{12}$} & 3 & 18 & 7 & 1 & 10 \\
\hline & 4 & 18 & 4 & 0 & 14 \\
\hline & 5 & 1 & 1 & 0 & 0 \\
\hline \multirow[t]{3}{*}{$\mathrm{M}_{22}$} & 3 & 25 & 5 & 8 & 12 \\
\hline & 4 & 16 & 5 & 4 & 7 \\
\hline & 5 & 9 & 0 & 1 & 8 \\
\hline \multirow[t]{3}{*}{$\mathbf{M}_{23}$} & 3 & 13 & 5 & 1 & 7 \\
\hline & 4 & 21 & 5 & 2 & 14 \\
\hline & 5 & 10 & 2 & 1 & 7 \\
\hline \multirow[t]{2}{*}{$\mathrm{J}_{1}$} & 3 & 1 & 0 & 0 & 1 \\
\hline & 4 & 2 & 1 & 0 & 1 \\
\hline \multirow[t]{2}{*}{$\mathrm{J}_{2}$} & 3 & 19 & 8 & 3 & 8 \\
\hline & 4 & 3 & 1 & 0 & 2 \\
\hline $\mathrm{J}_{3}$ & 3 & 17 & 5 & 8 & 4 \\
\hline \multirow[t]{4}{*}{ HS } & 3 & 30 & 8 & 5 & 17 \\
\hline & 4 & 41 & 8 & 1 & 32 \\
\hline & 5 & 8 & 1 & 0 & 7 \\
\hline & 6 & 1 & 0 & 0 & 1 \\
\hline
\end{tabular}


In Table 2, we cite the references for the geometries that satisfy the (IP) $)_{3}$, and those that satisfy the (WIP) $)_{3}$ but not the (IP) $)_{3}$. These numbers refer to the papers cited above for the corresponding groups. For instance, for the Mathieu group $\mathrm{M}_{22}$, geometries 3.1, 3.3, 3.6, 3.12 and 3.24 in [18] satisfy the (IP) $)_{3}$.

Tables 3 and 4 summarize the results for the $(\mathrm{IP})_{4}$ and $(\mathrm{WIP})_{4}$ properties in the same way; likewise, Tables 5 and 6 refer to the properties (IP) 5 and (WIP)5). Finally, Tables 7 and 8 summarize the results for the (IP) and (WIP) properties in the same way.

Since there is only one geometry of rank 6 among those that we looked at, we did not compile a table for the (IP) 6 and the (WIP) 6 . This geometry, which is number 6.1 for HS, is not $(\mathrm{WIP})_{6}$.

Table 2: References for geometries that are (IP $)_{3}$, and those that are (WIP $)_{3}$ but not (IP) $)_{3}$.

\begin{tabular}{|c|c|c|}
\hline Group & $(\mathrm{IP})_{3}$ & $(\mathrm{WIP})_{3}$ but not $(\mathrm{IP})_{3}$ \\
\hline \multirow[t]{2}{*}{$\mathrm{M}_{11}[14]$} & $3.3,3.5,3.11,3.12,3.13,3.18$ & 3.4 \\
\hline & $4.1,4.4$ & - \\
\hline \multirow[t]{3}{*}{$\mathrm{M}_{12}[12]$} & $3.2,3.4,3.5,3.7,3.9,3.16,3.19$ & 3.6 \\
\hline & $4.9,4.12,4.19,4.20$ & - \\
\hline & 5.1 & - \\
\hline \multirow[t]{4}{*}{$\mathrm{M}_{22}[18]$} & 3.1, 3.3, 3.6, 3.12, & $3.7,3,8,3.11,3.13$ \\
\hline & 3.24 & $3.17,3.18,3.23,3.25$ \\
\hline & $4.3,4.9,4.14,4.15,4.16$ & $4.8,4.11,4.12,4.13$ \\
\hline & - & 5.2 \\
\hline \multirow[t]{3}{*}{$\mathrm{M}_{23}[20]$} & $3.2,3.3,3.5,3.6,3.9$ & 3.13 \\
\hline & $4.2,4.4,4.8,4.17,4.19$ & $4.11,4.12$ \\
\hline & $5.3,5.9$ & 5.8 \\
\hline \multirow[t]{2}{*}{$\mathrm{J}_{1}[\mathbf{1 5}]$} & - & - \\
\hline & 4.1 & - \\
\hline \multirow[t]{2}{*}{$\mathrm{J}_{2}[17]$} & $3.1,3.2,3.3,3.4,3.9,3.16,3.17,3.18$ & $3.7,3.8,3.10$ \\
\hline & 4.2 & - \\
\hline \multirow[t]{2}{*}{$\mathrm{J}_{3}[19]$} & $3.2,3.3,3.4,3.7,3.9$ & $3.1,3.5,3.6,3.10$ \\
\hline & - & $3.13,3.14,3.15,3.17$ \\
\hline \multirow[t]{4}{*}{ HS [21] } & $3.2,3.4,3.7,3.13,3.14,3.19,3.24,3.25$ & $3.1,3.10,3.20,3.28,3.29$ \\
\hline & $4.1,4.3,4.5,4.7,4.8,4.14,4.15,4.16$ & 4.40 \\
\hline & 5.6 & - \\
\hline & - & - \\
\hline
\end{tabular}


Table 3: Number of geometries that are $(\mathrm{IP})_{4},(\mathrm{WIP})_{4}$ but not $(\mathrm{IP})_{4}$, and not $(\mathrm{WIP})_{4}$.

\begin{tabular}{l|crccc}
\hline Group & Rank & \# of geometries & $(\mathrm{IP})_{4}$ & $(\mathrm{WIP})_{4}$ but not $(\mathrm{IP})_{4}$ & not $(\mathrm{WIP})_{4}$ \\
\hline $\mathrm{M}_{11}$ & 4 & 11 & 2 & 0 & 9 \\
$\mathrm{M}_{12}$ & 4 & 18 & 3 & 0 & 15 \\
& 5 & 1 & 1 & 0 & 0 \\
$\mathrm{M}_{22}$ & 4 & 16 & 3 & 1 & 12 \\
& 5 & 9 & 0 & 1 & 8 \\
$\mathrm{M}_{23}$ & 4 & 21 & 3 & 1 & 17 \\
& 5 & 10 & 1 & 1 & 8 \\
$\mathrm{~J}_{1}$ & 4 & 2 & 1 & 0 & 1 \\
$\mathrm{~J}_{2}$ & 4 & 3 & 1 & 0 & 2 \\
$\mathrm{HS}$ & 4 & 41 & 5 & 0 & 36 \\
& 5 & 8 & 1 & 0 & 7 \\
& 6 & 1 & 0 & 0 & 1 \\
\hline
\end{tabular}

Table 4: References for geometries that are (IP) $)_{4}$, and those that are (WIP) $)_{4}$ but not (IP) $)_{4}$

\begin{tabular}{l|cc}
\hline Group & $(\mathrm{IP})_{4}$ & $(\mathrm{WIP})_{4}$ but not $(\mathrm{IP})_{4}$ \\
\hline $\mathrm{M}_{11}[\mathbf{1 4}]$ & $4.1,4.4$ & - \\
$\mathrm{M}_{12}[12]$ & $4.9,4.19,4.20$ & - \\
& 5.1 & - \\
$\mathrm{M}_{22}[18]$ & $4.3,4.9,4.14$ & 4.11 \\
& - & 5.2 \\
$\mathrm{M}_{23}[\mathbf{2 0}]$ & $4.2,4.8,4.19$ & 4.11 \\
& 5.3 & 5.9 \\
$\mathrm{~J}_{1}[\mathbf{1 5}]$ & 4.1 & - \\
$\mathrm{J}_{2}[\mathbf{1 7}]$ & 4.2 & - \\
$\mathrm{HS}[21]$ & $4.1,4.3,4.7,4.8,4.14$ & - \\
& 5.6 & - \\
\hline
\end{tabular}

Table 5: Number of geometries that are $(\mathrm{IP})_{5},(\mathrm{WIP})_{5}$ but not (IP) $)_{5}$, and not $(\mathrm{WIP})_{5}$.

\begin{tabular}{l|ccccc}
\hline Group & Rank & \# of geometries & $(\mathrm{IP})_{5}$ & $(\mathrm{WIP})_{5}$ but not $(\mathrm{IP})_{5}$ & not $(\mathrm{WIP})_{5}$ \\
\hline $\mathrm{M}_{12}$ & 5 & 1 & 0 & 0 & 1 \\
$\mathrm{M}_{22}$ & 5 & 9 & 0 & 1 & 8 \\
$\mathrm{M}_{23}$ & 5 & 10 & 1 & 0 & 9 \\
$\mathrm{HS}$ & 5 & 8 & 0 & 1 & 7 \\
& 6 & 1 & 0 & 0 & 1 \\
\hline
\end{tabular}


We used a computer with a Pentium III Xeon processor at $2.0 \mathrm{Ghz}$ and 3 gigabytes of memory (Rimm at 800Mhz) under Red Hat Linux 7.0 and MAGMA 2.10. Table 9 gives, for a group $G$ and a rank $r$, the number $n$ of geometries that $G$ has (see the note above Table 1 above) and the time needed for a complete analysis of all the $n$ geometries of rank $r$ that $G$ has. By this we mean the time needed to determine which ones are $(\text { IP })_{i},(\mathrm{WIP})_{i}$ (for $i=3, \ldots r$ ), (IP) and (WIP).

Table 6: References for geometries that are (IP) $)_{5}$, and those that are (WIP) $)_{5}$ but not (IP) $)_{5}$

\begin{tabular}{l|cc}
\hline Group & $(\mathrm{IP})_{5}$ & $(\mathrm{WIP})_{5}$ but not $(\mathrm{IP})_{5}$ \\
\hline $\mathrm{M}_{12}[\mathbf{1 2}]$ & - & - \\
$\mathrm{M}_{22}[\mathbf{1 8}]$ & - & 5.2 \\
$\mathrm{M}_{23}[\mathbf{2 0}]$ & 5.3 & - \\
$\mathrm{HS}[\mathbf{2 1}]$ & - & 5.6 \\
& - & - \\
\hline
\end{tabular}

Table 7: Number of geometries that are (IP), (WIP) but not (IP) and not (WIP).

\begin{tabular}{|c|c|c|c|c|c|}
\hline Group & Rank & \# of geometries & (IP) & (WIP) but not (IP) & not (WIP) \\
\hline \multirow[t]{2}{*}{$\mathrm{M}_{11}$} & 3 & 13 & 6 & 1 & 6 \\
\hline & 4 & 11 & 2 & 0 & 9 \\
\hline \multirow[t]{3}{*}{$\mathrm{M}_{12}$} & 3 & 18 & 7 & 1 & 10 \\
\hline & 4 & 18 & 3 & 0 & 15 \\
\hline & 5 & 1 & 0 & 0 & 1 \\
\hline \multirow[t]{3}{*}{$\mathrm{M}_{22}$} & 3 & 25 & 5 & 8 & 12 \\
\hline & 4 & 16 & 3 & 1 & 12 \\
\hline & 5 & 9 & 0 & 1 & 8 \\
\hline \multirow[t]{3}{*}{$\mathrm{M}_{23}$} & 3 & 13 & 5 & 1 & 7 \\
\hline & 4 & 21 & 3 & 1 & 17 \\
\hline & 5 & 10 & 1 & 0 & 9 \\
\hline \multirow[t]{2}{*}{$\mathrm{J}_{1}$} & 3 & 1 & 0 & 0 & 1 \\
\hline & 4 & 2 & 1 & 0 & 1 \\
\hline \multirow[t]{2}{*}{$\mathrm{J}_{2}$} & 3 & 19 & 8 & 3 & 8 \\
\hline & 4 & 3 & 1 & 0 & 2 \\
\hline $\mathrm{J}_{3}$ & 3 & 17 & 5 & 8 & 4 \\
\hline \multirow[t]{4}{*}{ HS } & 3 & 30 & 8 & 5 & 17 \\
\hline & 4 & 41 & 5 & 0 & 36 \\
\hline & 5 & 8 & 0 & 1 & 7 \\
\hline & 6 & 1 & 0 & 0 & 1 \\
\hline
\end{tabular}


The results obtained for the sporadic groups tend to show either that the (IP) is too strong, or that it calls for another axiom. Indeed, it reduces the maximal rank for geometries of $\mathrm{M}_{11}$, $\mathrm{M}_{12}, \mathrm{M}_{22}$ and $\mathrm{HS}$ to 4 . This is somehow not intuitive - for instance, since $\mathrm{M}_{11}$ is a maximal subgroup of $\mathrm{M}_{12}$, it is therefore natural to expect the maximal rank of a geometry of $\mathrm{M}_{11}$ to be less than the maximal rank for $\mathrm{M}_{12}$. The same holds for $\mathrm{M}_{22}$ and HS. On the other hand, the (IP) seems to be a good property in the sense that it 'kills' a lot of geometries, and therefore reduces the number of geometries that are obtained for a given group. In any case, we consider that it is still too early to decide whether or not to include the (IP) or one of its weakenings in the set of axioms of [9]. We recall the property $(2 T)_{1}$, which asks that the stabilizer of a flag $F$ of corank 1 in a geometry $\Gamma$ act two-transitively on the residue $\Gamma_{F}$ of $F$. Buekenhout, Dehon and Leemans started to test this property as early as in 1993, but they decided (together with Cara) to include it in the set of axioms only in 2000.

Table 8: References for geometries that are (IP), and those that are (WIP) but not (IP).

\begin{tabular}{|c|c|c|}
\hline Group & (IP) & (WIP) but not (IP) \\
\hline \multirow[t]{2}{*}{$\mathrm{M}_{11}[14]$} & $3.3,3.5,3.11,3.12,3.13,3.18$ & 3.4 \\
\hline & $4.1,4.4$ & - \\
\hline \multirow[t]{3}{*}{$\mathrm{M}_{12}[12]$} & $3.2,3.4,3.5,3.7,3.9,3.16,3.19$ & 3.6 \\
\hline & $4.9,4.19,4.20$ & - \\
\hline & - & - \\
\hline \multirow[t]{4}{*}{$\mathrm{M}_{22}[\mathbf{1 8}]$} & $3.1,3.3,3.6,3.12,3.24$ & $3.7,3,8,3.11,3.13$ \\
\hline & - & $3.17,3.18,3.23,3.25$ \\
\hline & $4.3,4.9,4.14$ & 4.11 \\
\hline & - & 5.2 \\
\hline \multirow[t]{3}{*}{$\mathrm{M}_{23}[20]$} & $3.2,3.3,3.5,3.6,3.9$ & 3.13 \\
\hline & $4.2,4.8,4.19$ & 4.11 \\
\hline & 5.3 & - \\
\hline \multirow[t]{2}{*}{$\mathrm{J}_{1}[15]$} & - & - \\
\hline & 4.1 & - \\
\hline \multirow[t]{2}{*}{$\mathrm{J}_{2}[17]$} & $3.1,3.2,3.3,3.4,3.9,3.16,3.17,3.18$ & $3.7,3.8,3.10$ \\
\hline & 4.2 & - \\
\hline $\mathrm{J}_{3}[19]$ & $3.2,3.3,3.4,3.7,3.9$ & $\begin{array}{c}3.1,3.5,3.6,3.10,3.13 \\
3.14,3.15,3.17\end{array}$ \\
\hline \multirow[t]{4}{*}{$\mathrm{HS}[21]$} & $3.2,3.4,3.7,3.13,3.14,3.19,3.24,3.25$ & $3.1,3.10,3.20,3.28,3.29$ \\
\hline & $4.1,4.3,4.7,4.8,4.14$ & - \\
\hline & - & 5.6 \\
\hline & - & - \\
\hline
\end{tabular}




\section{Results on other almost simple groups}

We also tested our program on other almost simple groups. We make some observations on the results obtained.

For the Suzuki simple group Sz(8), there are 147 geometries of rank three that satisfy the axioms of [9] (see [16]). All these geometries are thin. Of these, only 27 satisfy the (IP), and 48 are (WIP) but not (IP). Therefore the (IP) seems to be a good axiom to use in order to reduce the number of thin geometries that we obtain. It took 12 seconds to obtain these results.

For a symmetric group $\operatorname{Sym}(n)$ with $n \leqslant 7$, we found with our programs that the only inductively minimal geometry of rank $n-1$ satisfying the (IP) is the one with a linear diagram. Cara and Leemans then proved in [10] that this is the case for any value of $n$.

Table 9: Computing times.

\begin{tabular}{|c|c|c|c|}
\hline Group & Rank & \# of geometries & Computing time \\
\hline \multirow[t]{2}{*}{$\mathrm{M}_{11}$} & 3 & 13 & $1.4 \mathrm{secs}$ \\
\hline & 4 & 11 & $6.5 \mathrm{secs}$ \\
\hline \multirow[t]{3}{*}{$\mathrm{M}_{12}$} & 3 & 18 & $6.6 \mathrm{secs}$ \\
\hline & 4 & 18 & $45.8 \mathrm{secs}$ \\
\hline & 5 & 1 & $7.3 \mathrm{secs}$ \\
\hline \multirow[t]{3}{*}{$\mathrm{M}_{22}$} & 3 & 25 & $30 \mathrm{secs}$ \\
\hline & 4 & 16 & 57 secs \\
\hline & 5 & 9 & $6 \mathrm{~min} 20 \mathrm{secs}$ \\
\hline \multirow[t]{3}{*}{$\mathrm{M}_{23}$} & 3 & 13 & $2 \min 45 \mathrm{secs}$ \\
\hline & 4 & 21 & $20 \mathrm{~min} 08 \mathrm{secs}$ \\
\hline & 5 & 10 & $2 \mathrm{~h} 47 \mathrm{~min} 19 \mathrm{secs}$ \\
\hline \multirow[t]{2}{*}{$\mathrm{J}_{1}$} & 3 & 1 & 0.4 secs \\
\hline & 4 & 2 & 13 secs \\
\hline \multirow[t]{2}{*}{$\mathrm{J}_{2}$} & 3 & 19 & $6.3 \mathrm{secs}$ \\
\hline & 4 & 3 & $5.3 \mathrm{secs}$ \\
\hline $\mathrm{J}_{3}$ & 3 & 17 & $12 \mathrm{~min} 50 \mathrm{secs}$ \\
\hline \multirow[t]{4}{*}{$\mathrm{HS}$} & 3 & 30 & $7 \mathrm{~min} 39 \mathrm{secs}$ \\
\hline & 4 & 41 & $5 \mathrm{~h} 16 \mathrm{~min} 31 \mathrm{secs}$ \\
\hline & 5 & 8 & $1 \mathrm{~h} 57$ mins $15 \mathrm{secs}$ \\
\hline & 6 & 1 & $2 \mathrm{~h} 15 \mathrm{~min} 17 \mathrm{secs}$ \\
\hline
\end{tabular}




\section{Programs}

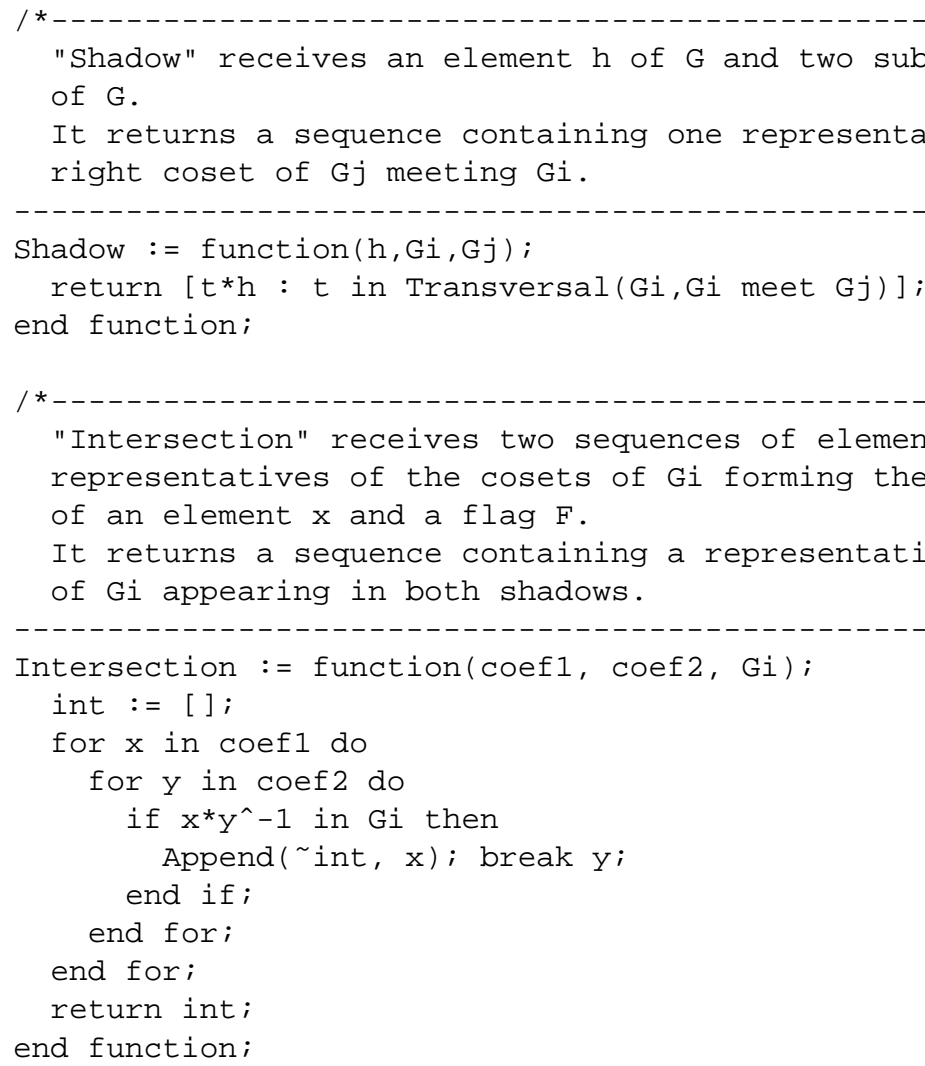
of an element $x$ and a flag $F$. of Gi appearing in both shadows.

"Intersection" receives two sequences of elements of $G$ that are representatives of the cosets of $\mathrm{Gi}$ forming the i-shadows

It returns a sequence containing a representative of each coset

"AreIncident" receives a set $x$ of subgroups of $G$, a set of indices corresponding to subgroups of $x$ having a right coset in the flag $F$, an element $h$ of $G$ such that $x[r]{ }^{*} h$ is a right coset of $\mathrm{F}$, a set $r 2$ of indices corresponding to the subgroups of $x$ having a right coset in the flag $F^{\prime}$ and an element $t$ of $G$ such that $x[r 2] * t$ is a right coset of $\mathrm{F}^{\prime}$.

It checks whether all elements of $F$ are incident to all elements of $\mathrm{F}^{\prime}$ or not.

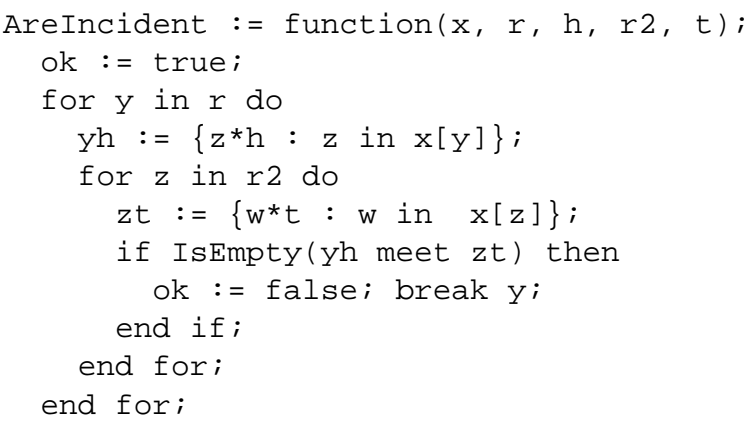


return ok;

end function;

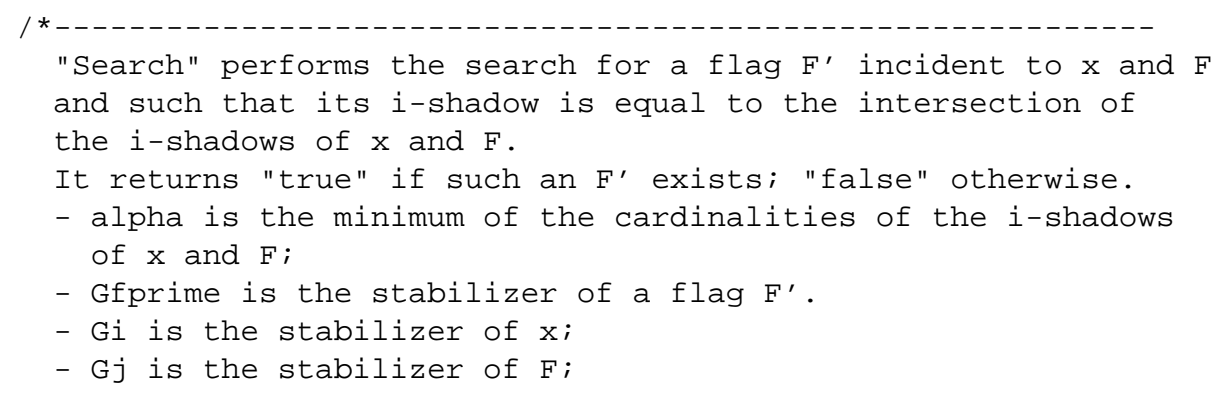

"Search" performs the search for a flag $F^{\prime}$ incident to $x$ and $F$ and such that its i-shadow is equal to the intersection of the $i$-shadows of $\mathrm{x}$ and $\mathrm{F}$.

It returns "true" if such an $\mathrm{F}^{\prime}$ exists; "false" otherwise.

- alpha is the minimum of the cardinalities of the i-shadows of $\mathrm{X}$ and $\mathrm{F}$;

- Geprime is the stabilizer of a flag $F^{\prime}$.

- Gi is the stabilizer of $x$;

- $\mathrm{Gj}$ is the stabilizer of $\mathrm{F}$;

Search := function(alpha, Gfprime, Gi, Gj, inte, $x, r, h, r 2)$; if alpha ne (\#Gfprime) / (\# (Gfprime meet Gi)) then return false; else

for $t$ in Transversal (Gi meet Gj, Gi meet $G j$ meet Gfprime) do shadowt := Shadow(t,Gfprime, Gi);

if \#Intersection(shadowt, inte, Gi) eq alpha then if AreIncident ( $x, r, h, r 2, t)$ then return true; end if; end if; end for; return false;

end if;

end function;

$/ \star$

"Property" checks whether, for a given type $i$ and a given element $G j$ of type $j$, for every flag $F$ such that the i-shadows of $\mathrm{G} j$ and $F$ have at least one element in common, there exists an $F^{\prime}$ such that $F^{\prime}$ is incident to $x$ and $F$ and the i-shadow of $F^{\prime}$ is the intersection of the $i$-shadows of $x$ and $F$.

- $k$ is a set of subsets of the set of types of the geometry; These subsets of types are the types that the flags $F$ may have.

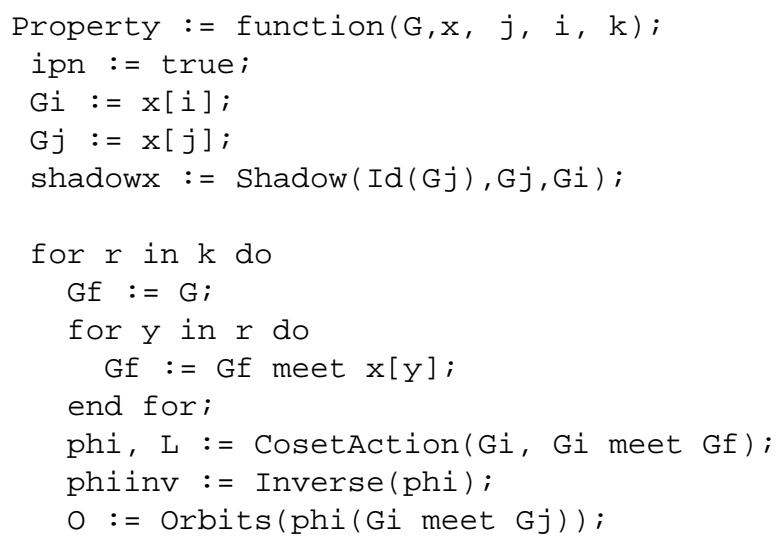




\section{An algorithmic analysis of the intersection property}

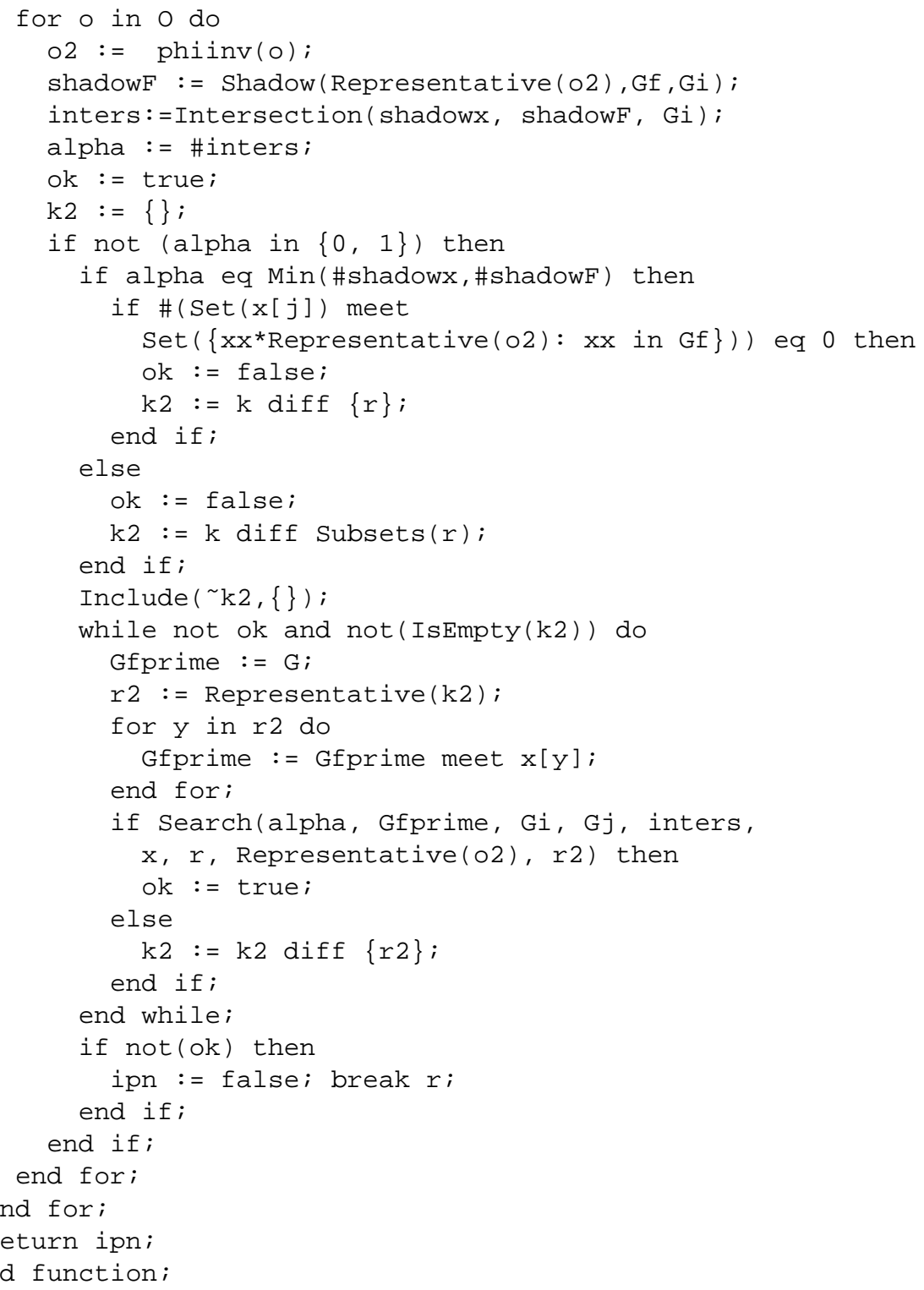

"IPn" receives a CosetGeometry $\mathrm{cg}$ and an integer $\mathrm{n}$ and checks whether cg satisfies the properties (IP) _n and (WIP) _n.

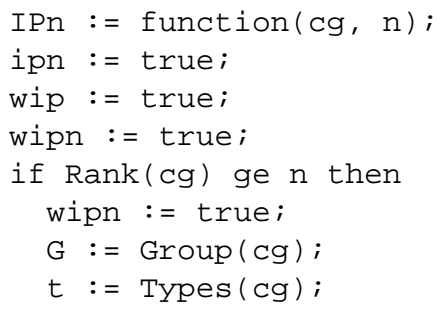




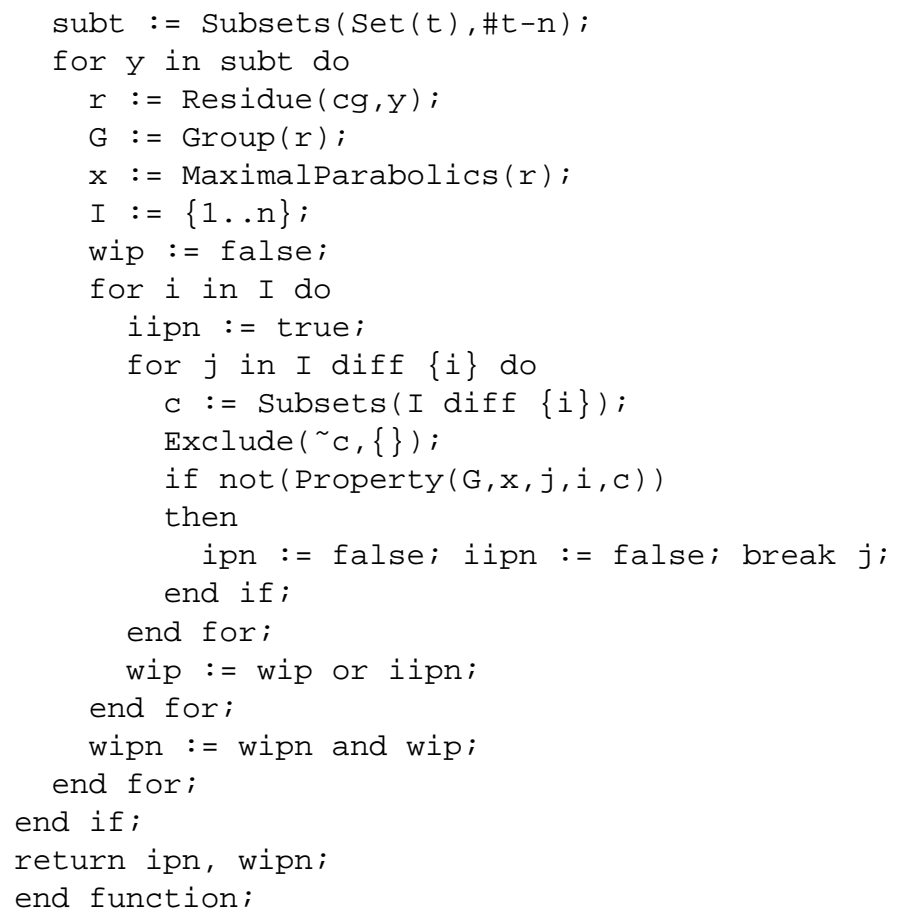

Acknowledgements. We gratefully acknowledge financial support for this project from the Belgian National Fund for Scientific Research.

\section{Appendix A.}

This appendix contains the source code of the paper (Section 6) as a text file. This file can be loaded in MAGMA using the 'load' command, and is available at

$$
\text { http://www.lms.ac.uk/jcm/7/lms2004-015/appendix-a. }
$$

Appendix B.

This appendix comprises a gzipped tar archive of text files that contain the maximal parabolic subgroups of the geometries for the eight smallest sporadic groups mentioned in Section 4. The archive also contains a README file, explaining how to use the files in Magma, and is available at

http://www.lms.ac.uk/jcm/7/lms2004-015/appendix-b.

\section{References}

1. M. Biliotti and A. Pasini, 'Intersection properties in geometry', Geom. Dedicata 13 (1982) 257-275. 284

2. W. Bosma, J. Cannon and C. Playoust, 'The Magma algebra system I: the user language', J. Symbolic Comput. 3/4 (1997) 235-265. 285 
3. F. Buekenhout, 'Diagrams for geometries and groups', J. Combin. Theory Ser. A 27 (1979) 121-151. 284, 285

4. F. Buekenhout, 'On the geometry of diagrams', Geom. Dedicata 8 (1979) 253-257. 284

5. F. Buekenhout, 'Separation and dimension in a graph', Geom. Dedicata 8 (1979) 297-298. 284

6. F. Buekenhout, 'The basic diagram of a geometry', Geometries and groups, Lecture Notes in Math. 893 (ed. M. Aigner and D. Jungnickel, Springer, Berlin, 1981) 1-29. 284

7. F. Buekenhout, 'The geometry of the finite simple groups', Buildings and the geometry of diagrams, Lecture Notes in Math. 1181 (ed. L. A. Rosati, Springer, Berlin, 1986) 1-78. 284

8. F. Buekenhout and A. PAsini, 'Finite diagram geometry extending buildings', Handbook of incidence geometry: buildings and foundations (North-Holland, Amsterdam, 1995) 1143-1254. 285

9. F. Buekenhout, P. Cara, M. Dehon and D. Leemans, 'Residually weakly primitive geometries of small almost simple groups: a synthesis', Topics in diagram geometry, Quaderni Mat. 12 (ed. A. Pasini, Department of Mathematics, Seconda Universita di Napoli, Caserta, 2003) 1-27. 284, 288, 292, 293

10. P. CARA and D. LeEmans, 'On inductively minimal geometries that satisfy the intersection property', preprint, Université Libre de Bruxelles, 2004,

http: / / cso.ulb.ac.be/ dleemans/abstracts/imgip.html. 293

11. M. Dehon, 'Classifying geometries with Cayley', J. Symbolic Comput. 17 (1994) 259-276. 284

12. M. Dehon and D. Leemans, Supplement to: 'Constructing coset geometries with MAGMA: an application to the sporadic groups $\mathrm{M}_{12}$ and $\mathrm{J}_{1}$ ', 2001, http: / / cso.ulb.ac.be/ dleemans/abstracts/algo.html. 288, 289, 290, 291, 292

13. M. Dehon and D. Leemans, 'Constructing coset geometries with Magma: an application to the sporadic groups $\mathrm{M}_{12}$ and $\mathrm{J}_{1}$ ', Atti Sem. Mat. Fis. Univ. Modena L (2002) 415-427. 284

14. M. Dehon, D. LeEmans and X. Miller, 'The residually weakly primitive and (IP) $)_{2}$ geometries of $\mathrm{M}_{11}$ ', preprint, Université Libre de Bruxelles, 1996,

http: / / cso.ulb.ac.be/ d deemans/abstracts/m11rwpri.html. 288, 289, 290, 292

15. H. Gottschalk and D. Leemans, 'The residually weakly primitive geometries of the Janko group $\mathrm{J}_{1}$ ', Groups and geometries (ed. A. Pasini et al., Birkhäuser, Basel, 1998) 65-79. 288, 289, 290, 292

16. D. LEEMANS, 'The residually weakly primitive geometries of the Suzuki simple group Sz(8)', Groups St Andrews 1997, II, London Math. Soc. Lect. Note Ser. 261 (ed. C. M. Campbell et al., Cambridge Univ. Press, Cambridge, 1999) 517-526. 293

17. D. LeEmans, Supplement to: 'The residually weakly primitive geometries of $\mathbf{J}_{2}$ ', 2001, http://cso.ulb.ac.be/ d dleemans/abstracts/j2.html.

289, 290, 292 
18. D. LeEmans, Supplement to: 'The residually weakly primitive geometries of $\mathbf{M}_{22}$ ', 2001, http: / / cso.ulb.ac.be/ dleemans/abstracts/m22.html. 288, 289, 290, 291, 292

19. D. LeEmans, Supplement to: 'The residually weakly primitive geometries of $\mathbf{J}_{3}$ ', 2003, http: / / cso.ulb.ac.be/ d dleemans/abstracts/j3.html. 288, 289, 292

20. D. Leemans, Supplement to: 'The residually weakly primitive geometries of $\mathrm{M}_{23}$ ', 2003, http://cso.ulb.ac.be/ d deemans/abstracts/m23.html. 288, 289, 290, 291, 292

21. D. LeEmans, Supplement to: 'The residually weakly primitive geometries of HS', 2003, http: / / cso.ulb.ac.be/ dleemans/abstracts/hs.html. 288, 289, 290, 291, 292

22. A. Pasini, Diagram geometries (Oxford Univ. Press, 1994). 284, 285

23. J. Tits, 'Les groupes de Lie exceptionnels et leur interprétation géométrique', Bull. Soc. Math. Belg. 8 (1956) 48-81. 284

24. J. Tits, 'Géométries polyédriques et groupes simples', Atti 2 a Riunione Groupem. Math. Express. Lat. Firenze (1962) 66-88. 286

Pascale Jacobs the.jackeswing.be

Dimitri Leemans dleemans@ulb.ac.be

http://cso.ulb.ac.be/ dleemans/

Université Libre de Bruxelles

Département de Mathématique

C.P. 216 - Géométrie

Boulevard du Triomphe

B-1050 Bruxelles

Belgium 